\title{
Short-form Mini-Nutritional Assessment with either BMI or calf circumference is effective in rating the nutritional status of elderly Taiwanese - results of a national cohort study
}

\author{
Alan C. Tsai ${ }^{1,2 *}$, Tsui-Lan Chang ${ }^{3,4}$ and Jiun-Yi Wang ${ }^{1}$ \\ ${ }^{1}$ Department of Healthcare Administration, Asia University, 500 Liufeng Road, Wufeng, Taichung 41354, Taiwan, ROC \\ ${ }^{2}$ Department of Health Services Management, School of Public Health, China Medical University, Taichung 404, \\ Taiwan, ROC \\ ${ }^{3}$ Department of Nursing, Hsin Yung Ho Hospital, Taoyuan 324, Taiwan, ROC \\ ${ }^{4}$ Department of Nursing, Hsin Sheng College of Medical Care and Management, Taoyuan 325, Taiwan, ROC
}

(Submitted 6 July 2012 - Final revision received 21 December 2012 - Accepted 12 January 2013 - First published online 25 February 2013)

\begin{abstract}
The present study was aimed to validate two normalised short-form (SF) Mini-Nutritional Assessments (MNA) that contained either BMI (Taiwan version 1, T1) or calf circumference (CC; Taiwan version 2, T2) for rating the nutritional status of elderly Taiwanese. Both versions adopted Taiwanese anthropometric cut-offs, but T2 further had the BMI item replaced by CC. We compared the ability of the two SF in rating the nutritional status of 2674 elderly Taiwanese in the 1999 'Taiwan Longitudinal Survey on Aging' with their respective full versions. We evaluated the agreement between the SF and full scales with weighted $\kappa$ and performed Cox regression analysis for the follow-up 4-year mortality according to nutritional status rated at baseline. The results showed that the agreements between the respective SF and the full MNA were good (weighted $\kappa: 0.679$ for T1 and 0.667 for T2). Both SF performed well in predicting follow-up 4-year mortality relative to the full MNA. In conclusion, MNA-T1-SF and -T2-SF have good consistency with the full MNA and have quite comparable abilities in rating the nutritional status of elderly Taiwanese. Both SF versions appear appropriate for functioning as stand-alone units for rating the nutritional status of the elderly in community-living settings or the general population.
\end{abstract}

Key words: Mini-Nutritional Assessment: Nutritional assessment: Malnutrition: Elderly: Taiwan

Nutrition is a key element in geriatric health. Undernutrition or protein-energy malnutrition is prevalent among the elderly. Studies have shown that approximately $10-15 \%$ of the general elderly population or community-living elderly are malnourished or at risk of malnutrition ${ }^{(1,2)}$. Rates are higher for the elderly in various living settings: $30-50 \%$ for the elderly in care centres; about $80 \%$ in nursing homes; up to $100 \%$ in hospitalised patients ${ }^{(3,4)}$. Malnutrition generally develops inconspicuously and if not stopped early in time, it can have serious consequences ranging from weight loss, general weakness, fatigue, anaemia, weakening of the immune system, increased susceptibility to diseases and infections, functional impairment, cognitive decline and even death. To stop this vicious cycle, early detection is crucial. To enable early detection, routine screening is necessary. However, to be used routinely, the tool must be simple, non-time-consuming and friendly to the users, and gentle and non-invasive to patients. It should not cause much pain, discomfort, suffering or great inconvenience to patients. Most importantly, diagnosis must be valid and reliable.

Although there are several geriatric nutrition assessment tools, few meet all these conditions. The Mini-Nutritional Assessment (MNA) is one that is closely meeting all these conditions. The MNA is one of the most widely used nutritional screening/assessment tools. It was developed in the $1990 \mathrm{~s}$ by Guigoz et al. ${ }^{(5,6)}$ based on the clinical data of Western elderly. The MNA consists of eighteen items and evaluates anthropometric, dietary, global and self-rated aspects of nutritional status; it normally takes about 10-20 min to administer, but the process could be much longer if the subject is frail or bedridden. This lengthy feature, to some degree, impedes its usefulness. To save rating time, Rubenstein et al. $^{(7)}$ restructured it into a two-part and two-stage tool. The first part is the short form (SF) that consists of six items and serves as a pre-screening unit. Those who are pre-screened as possible malnutrition are further evaluated with twelve additional

Abbreviations: CC, calf circumference; MNA, Mini-Nutritional Assessment; SF, short form; T1, Taiwanese version 1; T2, Taiwanese version 2. 
items (the rest of the full scale) to confirm the diagnosis. The SF generally predicts the full MNA well ${ }^{(4)}$. Recently, Kaiser et $a l^{(8)}$ have further proposed to use the MNA-SF as a stand-alone unit. In addition to rating the nutritional status, the MNA-SF also predicts the follow-up mortality risk of hospitalised or institutionalised elderly ${ }^{(9,10)}$. Since the MNA was developed for grading the nutritional status of Western elderly, modification would be necessary, especially regarding anthropometric and dietary items ${ }^{(11-13)}$, if the tool is to be applied to a non-Western population ${ }^{(14,15)}$.

Thus, we have recently modified the MNA-SF for the Taiwanese elderly (Taiwanese version 1, T1) by adopting population-specific anthropometric cut-off points. We have also further developed an alternative MNA-SF (Taiwanese version 2, T2) by replacing calf circumference (CC) for the BMI item $^{(2,4)}$. However, the abilities of these SF in rating the nutritional status of the elderly have not been robustly tested in large population-based samples. Since a simple, reliable and non-invasive tool is crucial for identifying the elderly who are at risk of malnutrition, we undertook the present study to validate the two versions of MNA-SF in a large national sample of elderly Taiwanese.

\section{Methods}

\section{Source of data}

The source of data was the 'Taiwan Longitudinal Survey on Aging', a population-based longitudinal cohort study conducted by the Bureau of Health Promotion of Taiwan. The study sample was drawn with a multistage national probability sampling process in $1989^{(16)}$. The first stage was to stratify the Taiwanese population into 361 primary sampling units and from which to randomly select fifty-six primary sampling units for further sampling, The second stage was proportional-to-size random selections of blocks (lins, the smallest administrative unit) from each of the selected primary sampling units, and the final stage was random selections of two eligible persons from each of the selected blocks. The process selected 4412 men and women, $\geq 60$ years old in 1989, to serve as the initial cohort for a longitudinal study. In order to maintain and further extend the younger age range of the study cohort, a second sample of 2462 subjects, 50-66 years old, was drawn from the population with the same procedure and added to the original cohort in 1996. The original or the combined cohort has undergone a series of six surveys to date (including 1989, 1993, 1996, 1999, 2003 and 2007).

In each survey, trained interviewers performed face-to-face interviews with a structured questionnaire on each subject at the participant's residence for eliciting demographic, socioeconomic, lifestyle and health- or healthcare-related data. A research institute trained all interviewers to ensure good consistency in interview skills. All components of the questionnaire were pre-tested and field-tested to ensure the validity and reliability before data collection. The completion rate of the survey ranged from $79 \cdot 1$ to $91 \cdot 8 \%$. The 1999 survey had expanded coverage on diet and nutrition, and included a nutritional assessment with the MNA administered by the interviewer. Therefore, it was appropriate for the present study. The dataset included all MNA items, except fluid intake. The survey from which the present study was based on was conducted according to the guidelines laid down in the Declaration of Helsinki and all procedures involving human subjects were reviewed and approved by governmentappointed representatives. Written informed consent was obtained from all subjects. Further detail of the sampling process has been described elsewhere ${ }^{(10)}$. The Bureau has released datasets of all six waves of the study for academic research. The present study analysed the data of 2674 participants who were $\geq 65$ years of age in 1999 .

\section{Mini-Nutritional Assessment}

We have recently developed two modified MNA versions for the Taiwanese. MNA-T1 adopted the Taiwanese-specific anthropometric cut-offs (items F, Q and R) and food patterns (items $\mathrm{K}$ and $\mathrm{M})^{(17,18)}$. MNA-T2 further replaced mid-arm circumference and CC items for the BMI item (by redistributing one of the three BMI points to the mid-arm circumference and two points to the CC). So, items $\mathrm{Q}$ and R in MNA-T2 are two and three points, respectively, instead of one point each in the original MNA. For MNA-T2-SF, CC replaces BMI. Thus, MNA-T2 contains no BMI item and needs no weight or height measurement, a process that can be quite involved and with questionable accuracy in functionally impaired subjects. MNA-T2 has been shown to function at least as well as MNA-T1 ${ }^{(4,19)}$.

\section{Grading the nutritional status and follow-up mortality}

The nutritional status of each subject was rated with the full and MNA-T1-SF and -T2-SF. All items, except fluid intake, were available in the dataset. Therefore, the score of the full MNA was based on seventeen items (sixteen items in T2) totalling twenty-nine points rather than eighteen items and thirty points. However, the total score was proportionately adjusted on a full-score (thirty-point) basis. A total score $<17$ was classified as malnourished, $17-23.5$ as at risk of malnutrition and $>23.5$ as normal ${ }^{(5)}$. The grading ability of the full scales of MNA-T1 and -T2 has been validated in elderly Taiwanese living in various settings ${ }^{(4)}$. Thus, these full scales were used as the criterion scales in the present study. For the MNA-SF, data for all six items were available in the dataset. The MNA-SF has a maximum score of 14 , and a score $\leq 7$ was considered as malnourished, $8-11$ as at risk of malnutrition and $\geq 12$ as normal ${ }^{(8)}$.

In geriatric populations, malnutrition is a strong predictor of follow-up mortality ${ }^{(9,10)}$. The MNA has also been shown to predict follow-up mortality in the elderly living in various settings. Lack of any better nutritional reference standard, we used the follow-up 4-year mortality as an external reference. Mortality data were taken from records gathered by the survey and confirmed by the data maintained by the National Household Registry and the National Health Insurance System. 


\section{Statistical analysis}

All statistical analyses were performed with the SPSS/Windows 15.0 software package (SPSS, Inc.). Descriptive data are expressed as $n$ and percentages or means and standard deviations. The nutritional status of each subject was graded with the full and MNA-T1-SF and -T2-SF. Cross-classification and weighted $\kappa$ were performed to determine the agreement between the full and SF scales of the MNA. Cox regression analyses were performed to determine the association of follow-up 4-year relative mortality risk with the nutritional status graded with various versions of the MNA at baseline adjusted for age, sex and years of formal education. In the analysis, lengths of survival time were calculated according to the records for subjects who died during the follow-up 4 years, and taken 48 months as the censoring time for subjects who survived. All values were weighting-adjusted according to the survey design. Statistical significance for all analyses was set at $\alpha=0 \cdot 05$.

\section{Results}

Table 1 shows the characteristics of the subjects. The sample included more men (55\%) than women; 57\% were 65-74 years old, 37\% were 75-84 years old and the rest were over 85 years old; $23 \%$ were current smokers and $21 \%$ were past smokers; $22 \%$ drank alcohol $\geq 1$ time/week; roughly $4 \%$ were current betel-nut chewers and $4 \%$ were past chewers (betel quid is used as a psychoactive substance mainly by adult males in Southeastern Asia and it increases the risk of

Table 1. Characteristics of the subjects ( $n$ 2674) (Number of subjects and percentages)

\begin{tabular}{lrr}
\hline Parameters & $n$ & $\%$ \\
\hline Sex & & \\
$\quad$ Men & 1474 & $55 \cdot 1$ \\
$\quad$ Women & 1200 & $44 \cdot 9$ \\
Age (years) & & \\
$65-74$ & 1518 & $56 \cdot 8$ \\
$75-84$ & 988 & $36 \cdot 9$ \\
$\geq 85$ & 168 & $6 \cdot 3$ \\
Formal education (years) & & \\
$\quad \leq 6$ & 2093 & $78 \cdot 3$ \\
$7-9$ & 267 & $10 \cdot 0$ \\
$10-12$ & 164 & $6 \cdot 1$ \\
$\geq 12$ & 150 & $5 \cdot 6$ \\
Smoking habit & & \\
$\quad$ Non-smoker & 1507 & $56 \cdot 4$ \\
Current smoker & 609 & $22 \cdot 8$ \\
$\quad$ Past smoker & 558 & $20 \cdot 8$ \\
Alcohol drinking (times/week) & & \\
$\quad<1$ & 2092 & $78 \cdot 2$ \\
$\quad \geq 1$ & 582 & $21 \cdot 8$ \\
Betel nut-chewing habit & & \\
$\quad$ Non-chewer & 2448 & $91 \cdot 5$ \\
Current chewer & 115 & $4 \cdot 3$ \\
$\quad$ Past chewer & 111 & $4 \cdot 2$ \\
Routine exercise (times/week) & \\
0 & & \\
$1-2$ & 1025 & $38 \cdot 3$ \\
$\geq 3$ & 151 & $5 \cdot 7$ \\
$\quad$ * & 1498 & $56 \cdot 0$ \\
\hline Defined as $>30$ min of medium or heavy exercise. & \\
$\quad$ & &
\end{tabular}

oral cancer) ${ }^{(20)}$; and $56 \%$ had physical exercise $\geq 3$ times/week. Of the 2674 elderly, 500 died during the follow-up 4 years.

Table 2 shows the distribution of item scores graded with MNA-T1 and T2. With the exception of items F, Q and R, the two versions shared the same questions and scores. Among the SF items, roughly $10 \%$ of subjects had poor appetite and $16 \%$ lost weight during the last 3 months, nearly $8 \%$ had impaired mobility, slightly over $10 \%$ had neuropsychological problems and about $28 \%$ had less than desired BMI $\left(21 \mathrm{~kg} / \mathrm{m}^{2}\right)$ but only about $8 \%$ had less than desired CC. Among the non-SF items, about $15 \%$ had skin sores, $13 \%$ consumed less than desirable protein-rich foods, $19 \%$ self-rated poor nutrition or unsure about own nutritional status, $45 \%$ rated own health status not as good as peers and 6\% had less than desired mid-arm circumference. The full MNA-T1 rated $2.0,13.9$ and $84.0 \%$; the full MNA-T2 rated 1.6, 12.9 and $85.5 \%$; the MNA-T1-SF rated 3.6, 19.5 and 76.9\%; and the MNA-T2-SF rated $2 \cdot 8,18.3$ and $78.9 \%$ as malnourished, at risk of malnutrition and normal, respectively. Table 3 shows the cross-classifications of the nutritional status rated with the MNA-SF against that rated with the full MNA. Both versions showed good consistency between the SF and the full scales. The two versions had comparable agreement between the respective SF and the full scale (weighted $\kappa: 0.679$ for T1 and 0.677 for T2, both $P<0.001$ ). Only thirty-eight subjects were rated downward (rated as normal instead of as at risk of malnutrition or malnourished) by the MNA-T1-SF and forty-five by the MNA-T2-SF compared with the respective full MNA.

Fig. 1 shows the Cox regression analyses of the follow-up 4-year survival curves stratified by nutritional status rated with the full and MNA-T1-SF and -T2-SF, respectively, adjusted for age, sex and years of formal education. Hazard ratios were 1, 2.39 (95\% CI 1.95, 2.94) and 6.59 (95\% CI 4.69, 9.27) for the full MNA-T1; $1,1 \cdot 82$ (95\% CI 1.49, 2.22) and $4 \cdot 17$ (95\% CI $3 \cdot 10$, 5.62 ) for the MNA-T1-SF; $1,2.50$ (95\% CI 2.03, 3.08), and $8 \cdot 10$ (95\% CI $5 \cdot 67,11.57)$ for the full MNA-T2; and 1, $2 \cdot 10$ (95\% CI $1.73,2.56)$ and $5.70(95 \%$ CI $4 \cdot 16,7 \cdot 79)$ for the MNA-T2-SF, for the elderly rated as normal, at risk of malnutrition and malnourished, respectively (all $P<0 \cdot 001$ ). MNA-T2 (both full scale and SF) seems to have stronger ability in predicting follow-up mortality than MNA-T1.

\section{Discussion}

\section{Consistency between the short-form Mini-Nutritional Assessments and full Mini-Nutritional Assessments}

The results show that both MNA-T1-SF and MNA-T2-SF have good consistency with the respective full scales in classifying nutritional status and have good abilities in predicting the follow-up 4-year mortality risk in a large population-representative sample of elderly Taiwanese. Compared with the full MNA, the respective SF rated larger proportions of the elderly as malnourished or as at risk of malnutrition and both SF were as effective as the full MNA in predicting mortality during the follow-up 4 years. Nutritional classifications graded with the normalised SF are well supported by the follow-up 4-year mortality. 
Table 2. Item-score patterns of the Mini-Nutritional Assessment (MNA) Taiwan version 1 (T1) and Taiwan version 2 (T2) by 2674 elderly Taiwanese*

(Number of subjects and percentages)

\begin{tabular}{|c|c|c|c|c|c|}
\hline \multirow[b]{2}{*}{ Items and conditions (item score) } & \multirow[b]{2}{*}{ Scoring } & \multicolumn{2}{|c|}{ MNA-T1 } & \multicolumn{2}{|c|}{ MNA-T2 } \\
\hline & & $n$ & $\%$ & $n$ & $\%$ \\
\hline \multicolumn{6}{|l|}{ Screeningt } \\
\hline \multicolumn{6}{|l|}{ A. Declined food intake over the past 3 months (2) } \\
\hline Severe loss of appetite & 0 & 269 & $10 \cdot 1$ & \multirow{3}{*}{\multicolumn{2}{|c|}{ Same as $\mathrm{T} 1 \ddagger$}} \\
\hline Moderate loss of appetite & 1 & 3 & 0.1 & & \\
\hline No loss of appetite & 2 & 2402 & 89.8 & & \\
\hline \multicolumn{6}{|l|}{ B. Weight loss during last months (3) } \\
\hline Lost $\geq 3 \mathrm{~kg}$ body weight & 0 & 416 & $15 \cdot 5$ & & \\
\hline Does not know & 1 & 7 & 0.3 & & \\
\hline No & 3 & 2251 & 84.2 & & \\
\hline \multicolumn{6}{|l|}{ C. Mobility (2) } \\
\hline Bed or chair-bound & 0 & 63 & 2.4 & & \\
\hline Able to get out of the bed but not able to go out & 1 & 139 & $5 \cdot 2$ & & \\
\hline Can get out of the bed and go out & 2 & 2472 & 92.4 & & \\
\hline \multicolumn{6}{|c|}{ D. Suffered psychological stress over the past 3 months (2) } \\
\hline Yes & 0 & 277 & $10 \cdot 4$ & & \\
\hline No & 2 & 2397 & $89 \cdot 6$ & & \\
\hline \multicolumn{6}{|l|}{ E. Neuropsychological problems (2) } \\
\hline Severe dementia or depression & 0 & 3 & 0.1 & & \\
\hline Mild dementia & 1 & 53 & $2 \cdot 0$ & & \\
\hline No psychological problems & 2 & 2618 & 97.9 & & \\
\hline \multicolumn{6}{|l|}{ F. BMI $\left(\mathrm{kg} / \mathrm{m}^{2}\right)(3)$} \\
\hline MNA-T1 & & & & & \\
\hline$<17$ & 0 & 71 & $2 \cdot 6$ & & \\
\hline $17-19$ & 1 & 210 & 7.9 & & \\
\hline $19-21$ & 2 & 469 & 17.5 & & \\
\hline$>21$ & 3 & 1924 & $72 \cdot 0$ & & \\
\hline MNA-T2 (replaced by item R, $\mathrm{cm}$ ) (male/female) & & & & & \\
\hline$<28 /<25$ & 0 & & & 76 & $2 \cdot 8$ \\
\hline $28-29 / 25-26$ & 1 & & & 66 & 2.5 \\
\hline $29-30 / 26-27$ & 2 & & & 74 & $2 \cdot 8$ \\
\hline$\geq 30 / \geq 27$ & 3 & & & 2458 & 91.9 \\
\hline Assessment & & & & & \\
\hline G. Lives independently (1) & & & & & \\
\hline No & 0 & 45 & 1.7 & & \\
\hline Yes & 1 & 2629 & $98 \cdot 3$ & & \\
\hline H. Taking $>3$ prescribed drugs/d (1) & & & & & \\
\hline Yes & 0 & 114 & $4 \cdot 3$ & & \\
\hline No & 1 & 2560 & $95 \cdot 7$ & & \\
\hline I. Pressure sore (1) & & & & & \\
\hline Yes & 0 & 397 & $14 \cdot 8$ & & \\
\hline No & 1 & 2277 & $85 \cdot 2$ & & \\
\hline J. Eat full meals per d (2) & & & & & \\
\hline 1 & 0 & 4 & 0.1 & & \\
\hline 2 & 1 & 93 & 3.5 & & \\
\hline$>3$ & 2 & 2577 & $96 \cdot 4$ & & \\
\hline K. Consumption of protein-rich foods§ (1) & & & & & \\
\hline 0 or 1 'yes' & 0 & 72 & $2 \cdot 7$ & & \\
\hline 2 'yes' & 0.5 & 287 & $10 \cdot 7$ & & \\
\hline 3 'yes' & 1 & 2315 & $86 \cdot 6$ & & \\
\hline L. Fruit and vegetable intake ( $\geq 2$ servings/d) (1) & & & & & \\
\hline No & 0 & 212 & 7.9 & & \\
\hline Yes & 1 & 2462 & $92 \cdot 1$ & & \\
\hline M. Fluid intake (1) & $N A \|$ & & & & \\
\hline N. Mode of feeding (2) & & & & & \\
\hline Unable to eat without assistance & 0 & 42 & 1.5 & & \\
\hline Self-fed with some difficulty & 1 & 79 & 3.0 & & \\
\hline Self-fed without difficulty & 2 & 2553 & 95.5 & & \\
\hline O. Self view of nutritional status (2) & & & & & \\
\hline Malnourished & 0 & 224 & 8.4 & & \\
\hline Uncertain of status & 1 & 292 & $10 \cdot 9$ & & \\
\hline No nutritional problem & 2 & 2158 & $80 \cdot 7$ & & \\
\hline P. Self-rated health status compared with peers (2) & & & & & \\
\hline Not as good & 0 & 1217 & 45.5 & & \\
\hline Uncertain or unknown & 0.5 & 0 & 0 & & \\
\hline As good & 1 & 1326 & $49 \cdot 6$ & & \\
\hline Better & 2 & 131 & 4.9 & & \\
\hline
\end{tabular}


Table 2. Continued

\begin{tabular}{|c|c|c|c|c|c|}
\hline \multirow[b]{2}{*}{ Items and conditions (item score) } & \multirow[b]{2}{*}{ Scoring } & \multicolumn{2}{|c|}{ MNA-T1 } & \multicolumn{2}{|c|}{ MNA-T2 } \\
\hline & & $n$ & $\%$ & $n$ & $\%$ \\
\hline \multicolumn{6}{|c|}{ Q. Mid-arm circumference $(\mathrm{cm})$ (male/female) (1) } \\
\hline $\begin{array}{l}\text { MNA-T1 (1) } \\
<22 \cdot 5 /<21\end{array}$ & 0 & 95 & $3 \cdot 6$ & & \\
\hline $22 \cdot 5-23 \cdot 5 / 21-22$ & 0.5 & 76 & $2 \cdot 8$ & & \\
\hline$\geq 23 \cdot 5 / \geq 22$ & 1 & 2503 & $93 \cdot 6$ & & \\
\hline \multicolumn{6}{|l|}{ MNA-T2 (2) } \\
\hline$<22.5 /<21$ & 0 & & & 95 & 3.6 \\
\hline $22 \cdot 5-23 \cdot 5 / 21-22$ & 1 & & & 76 & $2 \cdot 8$ \\
\hline$\geq 23 \cdot 5 / \geq 22$ & 2 & & & 2503 & $93 \cdot 6$ \\
\hline \multicolumn{6}{|c|}{ R. Calf circumference $(\mathrm{cm})$ (male/female) } \\
\hline \multicolumn{6}{|c|}{ MNA-T1 (1) } \\
\hline$<28 /<25$ & 0 & 76 & $2 \cdot 8$ & & \\
\hline$\geq 28 / \geq 25$ & 1 & 2598 & $97 \cdot 2$ & & \\
\hline \multicolumn{6}{|l|}{ MNA-T2 (replacing item F) } \\
\hline $\begin{array}{l}\text { * The full MNA-T1 rated } 2.0,13.9 \text { and } \\
19.5 \text { and } 76.9 \% \text { and the MNA-T2 } \\
\text { respectively. } \\
\text { † The screening section (first six items) } \\
\text { † Items not indicated are the same as } \\
\text { § Item K - frequency of consumption, in } \\
\text { || Data not available in the dataset. }\end{array}$ & $\begin{array}{l}2.9 \text { and } 85 \\
\text { as malnour } \\
\mathrm{R} \text { in MNA- }\end{array}$ & $\begin{array}{l}\text {, the sh } \\
\text { d, at } r i\end{array}$ & orm $(\mathrm{S}$ & $\begin{array}{l}\text { UNA-T1 } \\
\text { ition anc }\end{array}$ & $\begin{array}{l}\text { od } 3.6 \text {, } \\
\text { ormal, }\end{array}$ \\
\hline
\end{tabular}

\section{Comparing with other studies}

According to Cox regression, MNA-T2 is at least as effective as MNA-T1 in predicting the follow-up 4-year mortality risk. Both full and MNA-T2-SF have slightly higher hazard ratios (and 95\% CI) than the respective T1 scales. This finding is somewhat different from that observed by Kaiser et al. ${ }^{(19)}$ who reported that CC-MNA-SF (equivalent to MNA-T2 in the present study) tended to underestimate nutritional risk compared with BMI-MNA-SF (equivalent to MNA-T1). A possible reason could be the differences in the scoring of item $\mathrm{R}$ (CC). Kaiser et $a l{ }^{(19)}$ used an all-or-none (either three points or none with a single cut-off point of $31 \mathrm{~cm}$ ) scoring system in CC-MNA-SF, whereas MNA-T2 used a graded scoring system (one additional point for each incremental centimetre for up to three points). Thus, subjects with marginal CC would get partial scores similar to item F (BMI) in MNA-T1.

\section{Short-form Mini-Nutritional Assessments compared well with the full Mini-Nutritional Assessments}

Thus, in principle, the MNA-SF can function as a stand-alone unit for screening/assessing the nutritional status. Recently, Kaiser et $a l .{ }^{(8)}$ found that the MNA-SF compared well with the full MNA by analysing studies conducted in many countries and proposed to revise the two-category MNA-SF into a three-category scale so that it could function as a stand-alone tool similar to the full MNA. In the present

Table 3. Cross-classification of the nutritional status graded with the full Mini-Nutritional Assessment (MNA) and the short-form (SF) MNA (n 2674)

\begin{tabular}{|c|c|c|c|c|c|c|}
\hline \multirow[b]{2}{*}{ According to the MNA-SF } & \multicolumn{4}{|c|}{ According to the full MNA } & \multirow[b]{2}{*}{ Weighted $\kappa$} & \multirow[b]{2}{*}{$95 \% \mathrm{Cl}$} \\
\hline & Malnourished & At risk of malnutrition & Normal & Total & & \\
\hline & Full MNA-T1 & & & & & \\
\hline \multicolumn{7}{|l|}{ MNA-T1 } \\
\hline Malnourished & 49 & 48 & 0 & $97(3.6)$ & $0.679^{* * *}$ & $0.647,0.711$ \\
\hline At risk of malnutrition & 5 & 287 & 228 & $520(19.5)$ & & \\
\hline Normal & 0 & 38 & 2019 & 2057 (76.9) & & \\
\hline \multirow[t]{2}{*}{ Total } & $54(2 \cdot 0)$ & $373(13 \cdot 9)$ & $2247(84 \cdot 0)$ & & & \\
\hline & Full MNA-T2 & & & & & \\
\hline \multicolumn{7}{|l|}{ MNA-T2 } \\
\hline Malnourished & 41 & 34 & 0 & $75(2 \cdot 8)$ & $0.677^{\star \star \star}$ & $0.633,0.702$ \\
\hline At risk of malnutrition & 3 & 266 & 219 & 488 (18.2) & & \\
\hline Normal & 0 & 45 & 2066 & $2111(78 \cdot 9)$ & & \\
\hline Total & $44(1 \cdot 6)$ & $345(12.9)$ & $2285(85.5)$ & & & \\
\hline
\end{tabular}

T1, Taiwan version 1; T2, Taiwan version 2. $* * \star P<0.001$. 

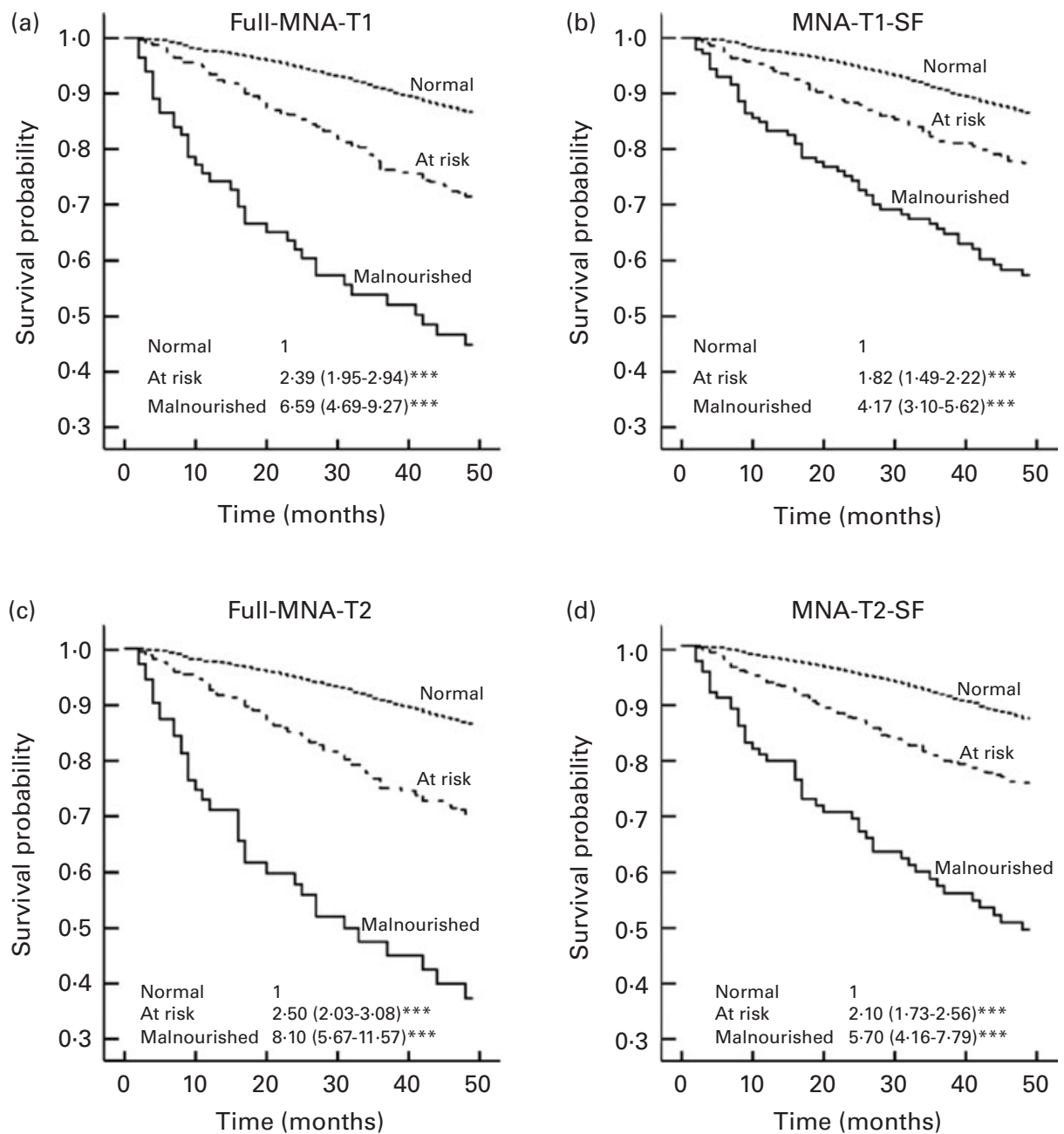

Fig. 1. Cox regression analyses of the follow-up 4-year survival curves stratified by nutritional status rated with the full and short-form (SF) versions of Taiwan version 1 (T1) and 2 (T2), respectively, adjusted for age, sex and years of formal education. Sets of data in each panel are hazard ratios (HR) and $95 \% \mathrm{Cl}$ for subjects rated as normal (reference), at risk of malnutrition and malnourished, respectively. (a) Full Mini-Nutritional Assessment (MNA)-T1: normal - HR 1, at risk of malnutrition - HR 2.39 (95\% Cl 1.95, 2.94, $P<0.001)$, malnourished - HR 6.59 (95\% Cl 4.69, 9.27, $P<0.001)$; (b) MNA-T1-SF: normal - HR 1, at risk of malnutrition - HR $1.82(95 \% \mathrm{Cl} 1.49,2.22, P<0.001)$, malnourished - HR 4.17 (95\% Cl 3.10, 5.62, $P<0.001)$; (c) full MNA-T2: normal - HR 1, at risk of malnutrition - HR 2.50 (95\% Cl 2.03, 3.08, P<0.001), malnourished - HR 8.10 (95\% Cl 5.67, 11.57, $P<0.001$ ); (d) MNA-T2-SF: normal - HR 1, at risk of malnutrition - HR $2.10(95 \% \mathrm{Cl} 1.73,2.56, P<0.001)$, malnourished - HR $5.70(95 \% \mathrm{Cl} 4.16,7.79, P<0.001)$. In the analysis, lengths of survival time were calculated according to the records maintained by the 'Taiwan Longitudinal Survey on Aging' project for subjects who died during the follow-up 4 years, and taken 48 months as the censoring time for subjects who survived.

study, we have seen that the normalised SF can function satisfactorily in classifying the nutritional status, and CC could substitute BMI and still at least preserve the rating ability of the scale.

\section{A stand-alone short-form Mini-Nutritional Assessments with calf circumference has advantages}

A stand-alone MNA-SF with CC clearly has many advantages. It is probably simpler and easier to measure CC than to measure weight and height, especially for the frail or bedridden elderly. The simplicity, brevity and non-invasiveness of the tool can make 'routine screening and timely intervention' an easier goal to fulfil in geriatric care. These features will also make the MNA-SF a more acceptable tool for screening or monitoring the nutritional status of the elderly at the community or population level. However, the applicability of the MNA-SF in persons on haemodialysis requires further investigation because the tool has been observed to underrate the risk of malnutrition in these persons ${ }^{(21)}$.

\section{Limitations of the study}

The present study has some limitations. (1) Ideally, the reference standard used to compare the predictive ability of the MNA scales should be a widely accepted nutritional indicator such as serum albumin. However, the 'Taiwan Longitudinal Survey on Aging' does not contain such data. Further confirmation of the present study results with a more robust nutritional indicator is desired. (2) The full MNA were based on seventeen, instead of eighteen, items due to the lack of water intake data in the dataset, which might lead to a slight 
underestimation of nutritional risk by the full scale. (3) Most data of the survey were collected by questionnaire interviews which generally have acceptable quality but shortcomings are inevitable. A feature of the present study is that the dataset is from a relatively large population-based sample. The present results should be applicable to populations that share the anthropometric and lifestyle characteristics of the Taiwanese.

\section{Conclusion}

The study has shown that both MNA-SF are appropriate for classifying the nutritional status of elderly Taiwanese. Both versions have high consistency with the full scales and have strong ability in predicting follow-up mortality which is a major variable reflecting the nutritional status. These results suggest that both MNA-SF can function as stand-alone units for screening the nutritional risk of elderly Taiwanese. We recommend both versions (T1 and T2) of this simple, time-efficient and noninvasive MNA-SF for 'routine screening' of the nutritional status of elderly Taiwanese living in most settings.

\section{Acknowledgements}

The present study is based on the data from the 'Taiwan Longitudinal Survey on Aging', provided by the Bureau of Health Promotion, Department of Health, ROC (Taiwan). Descriptions or conclusions herein do not represent the viewpoint of the Bureau. The study received no funding support from any source. A. C. T. conceived the idea and directed the study, and is the main writer of the manuscript; T.-L. C. performed the statistical analysis and reviewed the manuscript; J.-Y. W. directed the statistical analysis and reviewed the manuscript. All authors declare that they have no competing interests.

\section{References}

1. Tsai AC, Ho CS \& Chang MC (2008) Assessing the prevalence of malnutrition with the Mini Nutritional Assessment (MNA) in a nationally representative sample of elderly Taiwanese. J Nutr Health Aging 12, 239-243.

2. Tsai AC, Chang TL, Yang T-W, et al. (2010) A modified Mini Nutritional Assessment without BMI predicts nutritional status of community-living elderly in Taiwan. J Nutr Health Aging 14, 183-189.

3. Cereda E (2012) Mini Nutritional Assessment. Curr Opin Clin Nutr Metab Care 15, 29-41.

4. Tsai AC, Chang TL, Wang YC, et al. (2010) Populationspecific short-form Mini Nutritional Assessment with BMI or calf-circumference can predict risk of malnutrition in community-living or institutionalized elderly in Taiwan. $J \mathrm{Am}$ Dietet Assoc 110, 1328-1334.

5. Guigoz Y, Lauque S \& Vellas BJ (2002) Identifying the elderly at risk for malnutrition. The Mini Nutritional Assessment. Clin Geriatr Med 18, 737-757.
6. Vellas B, Guigoz Y, Garry PJ, et al. (1999) The Mini Nutritional Assessment (MNA) and its use in grading the nutritional state of the elderly patients. Nutrition 15, 116-122.

7. Rubenstein LZ, Harker JO, Salva A, et al. (2001) Screening for undernutrition in geriatric practice: developing the shortform Mini-Nutritional Assessment (MNA-SF). J Gerontol A Biol Sci Med Sci 56A, M366-M372.

8. Kaiser MJ, Bauer JM, Rämsch C, et al. (2009) Validation of the Mini Nutritional Assessment ${ }^{\circledR}$ Short-Form (MNA-SF): a practical tool for identification of nutritional status. J Nutr Health Aging 13, 782-788.

9. Persson MD, Brismar KE, Katzarski KS, et al. (2002) Nutritional status using Mini Nutritional Assessment and Subjective Global Assessment predict mortality in geriatric patients. J Am Geriatr Soc 50, 1996-2002.

10. Tsai AC, Yang SF \& Wang JY (2010) Validation of populationspecific Mini-Nutritional Assessment with its long-term mortality-predicting ability - results of a population-based longitudinal 4-year study in Taiwan. Br J Nutr 104, 93-99.

11. Deurenberg P, Deurenberg-Yap M \& Guricci S (2002) Asians are different from Caucasians and from each other in their body mass index/body fat percent relationship. Obes Res $\mathbf{3}$, $141-146$.

12. Stevens J (2003) Body mass index and mortality in Asian populations: implication for obesity cut-points. Nutr Rev 61, 104-107.

13. WHO expert consultation (2004) Appropriate body-mass index for Asian populations and its implications for policy and intervention strategies. Lancet 363, 157-163.

14. Chumlea WC (1999) The state of the Mini Nutritional Assessment? Nutrition 15, 159-161.

15. Chumlea WC (2006) Is the MNA valid in different populations and across practice settings? J Nutr Health Aging 10, 524-533.

16. Bureau of Health Promotion, Department of Health, Taiwan (1989) Survey of the Elderly in Taiwan. http://www.bhp. doh.gov.tw/BHPnet/Portal/Them.aspx?No=200712270002 (accessed 10 June 2011).

17. Tsai AC, Ho CS \& Chang MC (2007) Population-specific anthropometric cut-points improve the functionality of the Mini Nutritional Assessment (MNA) in elderly Taiwanese. Asia Pac J Clin Nutr 16, 656-662.

18. Tsai AC \& Ku PY (2008) Population-specific Mini Nutritional Assessment effectively predicts the nutritional state and follow-up mortality of institutionalized elderly Taiwanese regardless of cognitive status. Br J Nutr 100, 152-158.

19. Kaiser MJ, Bauer JM, Uter W, et al. (2011) Prospective validation of the modified Mini Nutritional Assessment shortforms in the community, nursing home, and rehabilitation settings. J Am Geriatr Soc 59, 2124-2128.

20. Reichart PA \& Warnakulasuriya S (2012) Oral lichenoid contact lesions induced by areca nut and betel quid chewing: a mini review. J Investig Clin Dent 3, 163-166.

21. Tsai AC \& Chang M-Z (2011) Long-form but not short-form Mini-Nutritional Assessment is appropriate for grading nutritional risk of patients on hemodialysis - a cross-sectional study. Int J Nurs Stud 48, 1429-1435. 